\title{
Improving teamwork learning in design education
}

\author{
Chong-Wen Chen ${ }^{1}$, Pei-Yu Lin ${ }^{2}$ \\ Yuan Ze University / Department of Information Communication
}

\begin{abstract}
Teamwork learning plays an important role in design education. Although many studies have shown how teamwork learning can enhance student motivation, few of them have focused on the schemes for making appropriate team composition and increasing open communication. In this paper, we apply game design elements and concepts such as unpredictability, curiosity, and feedback to foster students' creative thinking and engagement. We propose several principles for educators and conduct a case study of two design courses, the Introduction to Design Principles and the Creativity Techniques. Based on the questionnaire survey of student opinion and teacher's observations, we conclude that random grouping and diverse team compositions can bring more different value propositions and viewpoints, which is the key to creating novel design ideas. In addition, intensive group discussions with time restriction and immediate feedback such as result sharing, visual presentation and comments can help stimulate the effectiveness of peer learning and open communication.
\end{abstract}

Keywords: creativity; design thinking; engagement; gamification; peer learning 